\title{
Fibrogenesis in Kidney Transplant: Dysfunction Progress Biomarkers
}

\author{
J.S. Costa ${ }^{a, *}$, R. Alves ${ }^{a}$, V. Sousa ${ }^{b}$, C. Marinho ${ }^{b}$, C. Romãozinho ${ }^{a}$, L. Santos ${ }^{a}$, F. Macário ${ }^{a}$, J. Pratas $^{a}$, \\ L. Prado e Castro ${ }^{b}$, M. Campos ${ }^{a}$, and A. Figueiredo ${ }^{c}$

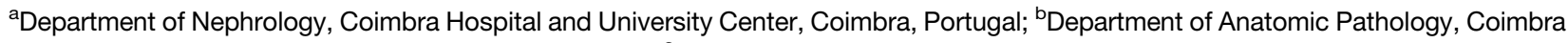 \\ Hospital and University Center, Coimbra, Portugal; and ${ }^{\mathrm{C} D e p a r t m e n t ~ o f ~ U r o l o g y ~ a n d ~ K i d n e y ~ T r a n s p l a n t a t i o n, ~ C o i m b r a ~ H o s p i t a l ~ a n d ~}$ \\ University Center, Coimbra, Portugal
}

\begin{abstract}
Introduction. Fibrogenesis markers, such as alpha-actin (AA), CD163 (macrophages), and E-cadherin, have been studied as chronic kidney allograft injury (CAI) predictors, a major cause of allograft failure.

Objective. Investigate the value of these markers in predicting CAI and initiation of dialysis.

Materials and Methods. Retrospective analysis of 26 kidney allograft biopsies (from 22 patients with CAI) during 2 years, evaluating intensity and percentage of marked cells on glomeruli and tubulointerstitial compartment. At the time of the biopsy, patients were $45.5 \pm 15.8$ years and 4.2 years after transplant, and they had a mean glomerular filtration rate (GFR) of $25.8 \pm 9.9 \mathrm{~mL} / \mathrm{min}$. From an average of 8.5 glomeruli per biopsy, there was $\leq 25 \%$ sclerosis in 17 cases, $26 \%$ to $50 \%$ in 5 , and $>50 \%$ in 4 . Interstitial fibrosis or tubular atrophy affected $\leq 25 \%$ of cortical area in 14 cases, $26 \%$ to $50 \%$ in 8 , and $>50 \%$ in 2. Twelve patients started dialysis $5.8 \pm 4.7$ years after transplant, with an average GFR $20.9 \mathrm{~mL} / \mathrm{min}$ at the time of the biopsy.

Results. There was a higher intensity and percentage of CD163-marked cells in the tubulointerstitial compartment in advanced interstitial fibrosis. We found an association between intensity of AA in the tubulointerstitial compartment and initiation of dialysis $(P=.003)$ and a negative correlation between intensity of E-cadherin loss and GFR $(r=-0.56, P=.012)$.

Conclusions. In our study, intensity of tubulointerstitial AA was shown to be a predictor of initiation of dialysis, and E-cadherin loss intensity was associated to CAI progression. However, prospective and larger studies are needed to evaluate the predictive value of these markers.
\end{abstract}

$\mathbf{T}$ HE KIDNEY responds to injuries (hemodynamic, immune, or metabolic) through adaptive changes that may evolve toward either its own healing and functional recovery or scarring with loss of the kidney function. Scarring is characterized by the replacement of intrinsic renal cells with fibrous tissue made of collagenous extracellular matrix (ECM) [1]. This affects the glomeruli, tubules, and interstitium and vessel.

Mesangial cells are located in the glomeruli and play a central role in the process of healing or scarring. They can differentiate into an embryonic phenotype and acquire alpha-smooth muscle actin (AA), allowing them to proliferate, to migrate, and to restore the glomerular structural integrity [1]. However, when uncontrolled mesangial proliferation occurs, an excessive matrix results in the "scar."

Some tubular cells respond to an acute injury through necrosis or apoptosis, whereas dedifferentiation into an embryonic phenotype and acquisition of AA allow surviving cells to attempt restoring tubule integrity. Repeated or sustained injury is likely to induce epithelial-mesenchymal

*Address correspondence to Joana Silva Costa, Centro Hospitalar e Universitário de Coimbra-Hospitais da Universidade de Coimbra, Serviço de Nefrologia, Praceta Prof Mota Pinto, 3000075 Coimbra, Portugal. E-mail: joana.c.s.costa@gmail.com

$0041-1345 / 17$

http://dx.doi.org/10.1016/j.transproceed.2017.01.063 
transition (EMT). During EMT, epithelial cells acquire migratory properties of mesenchymal cells and transit into the interstitium [2,3]. Loss of E-cadherin, a transmembrane glycoprotein and a differentiated epithelium marker, results in the epithelium disintegration and is an early event during EMT. Loss of epithelial cells contributes to myofibroblast formation and fibrosis [4].

Both glomerulosclerosis and tubulointerstitial fibrosis are stimulated by cytokines and growth factors. Transforming growth factor $\beta 1$ (TGF- $\beta 1$ ) is the most important growth factor inducing fibrosis [5]. Infiltration of the glomeruli and renal interstitium with inflammatory cells is also a common pathway in the pathogenesis of glomerulosclerosis and tubulointerstitial fibrosis. If chronic injury and inflammation persist, both M1 and M2 macrophages phenotypes can be found. M1 macrophages are the first to be recruited for removal of dead cells, but they can also exacerbate renal cell damage by means of their proinflammatory action $[2,6]$. Furthermore, M2 phenotypes are anti-inflammatory macrophages that can promote not only epithelial and vascular repair but also fibrosis [5,6]. Specific M2 macrophage markers CD163, CD206, and CD301 can be used to differentiate them through flow cytometry. Macrophage infiltration and local proliferation are related to the severity of glomerular and tubulointerstitial damages and serve as prognostic marker of disease progression [6]. Furthermore, proliferative macrophages and AA and myofibroblast infiltration in areas of severe renal damage suggest an association between macrophages and renal fibrosis in chronic kidney disease progression [6].

Concerning kidney transplants, one study concluded that AA expression in time-zero biopsies, especially its combination with interstitial fibrosis, could strongly predict chronic renal allograft injury (CAI) [7]. TGF- $\beta 1$ has been suggested as the centric hypothesis for chronic allograft rejection and CAI. Both immune (acute allograft rejection) and nonimmune factors (calcineurin inhibitors [CNIs], infection, ischemia, chronic hypertension, and others) can be responsible for the allograft damage, and the intrarenal overexpression of TGF- $\beta 1$ can promote a progressive damage $[8,9]$. However, TGF- $\beta 1$ is also a potent immunosuppressant in the kidney allograft. The use of TGF- $\beta 1$ as a selective blockage (blocking the fibrogenic arm but not the immunoregulatory arm) may be of benefit in minimizing the allograft's damage, but it remains a challenge [9].

It may be useful to understand fibrogenesis mechanisms and identify CAI predictors to its early diagnosis. We have designed this study to investigate the value of AA and CD163 expression as well as the E-cadherin loss in predicting CAI and initiation of dialysis.

\section{MATERIALS AND METHODS Population}

We retrospectively analyzed 26 kidney allograft biopsies from 22 patients with CAI whose transplantations were performed between January 2014 and December 2015. The 22 patients included 4 women and 18 men; 2 were living donor recipients and the rest deceased donor recipients. At the time of biopsy, their mean age was $45.5 \pm 15.8$ years (minimum 19 years, maximum 72 years). All patients were medicated with corticoids, 21 patients with CNIs, 20 patient with antimetabolite drugs, and 2 patients with mammalian target of rapamycin inhibitors. They presented an average of 4.2 years after transplant (minimum 4 years, maximum 18 years), and their mean glomerular filtration rate (GFR) was 25.8 $\pm 9.9 \mathrm{~mL} / \mathrm{min}$ (7 patients with GFR $30-60 \mathrm{~mL} / \mathrm{min}, 12$ patients with GFR 15-29 $\mathrm{mL} / \mathrm{min}$, and 3 patients with GFR $<15 \mathrm{~mL} / \mathrm{min}$ ). Twelve patients started dialysis $5.8 \pm 4.7$ years after transplant. In association with the diagnosis of CAI, 8 patients had acute T-cellmediated rejection (IA in 6 cases and IB in 2 cases), 11 patients had borderline changes, and 5 patients had histologic findings suggesting CNI toxicity.

From the previous report of the analyzed biopsy, with an average of 8.5 glomeruli per biopsy, glomerulosclerosis was detected in $\leq 25 \%$ of the glomeruli in 17 cases, $26 \%$ to $50 \%$ in 5 cases, and $>50 \%$ in 4 cases. At biopsy re-evaluation, 5 cases did not have glomeruli. There were 2 cases of polyomavirus nephropathy with interstitial fibrosis. Interstitial fibrosis or tubular atrophy affected $\leq 25 \%$ of cortical area in 14 cases, $26 \%$ to $50 \%$ in 8 , and $>50 \%$ in 2 cases.

\section{Evaluation of Marked Cell}

Using normal kidney tissue from a nephrectomized patient with a localized tumor as control, 2 pathologists evaluated the intensity and the percentage of marked cells (AA and CD163) in glomeruli, tubules, and interstitium and the loss of E-cadherin in the tubules. The final result was based on agreement or averaging of their results. The intensity of marked cell or loss of E-cadherin was established between 1 (low positive) and $3+$ (high positive), and the percentage of marked cell or loss of E-cadherin was defined from 0 to $100 \%$.

\section{Statistical Analysis}

Spearman correlation coefficient was used to assess the relationship between AA, CD163, and E-cadherin loss intensity and percentage with CAI. $\chi^{2}$ and Wilcoxon tests were used to assess the relationship between these markers' intensity and percentage, respectively, with dialysis. The value of the markers to predict CAI and the initiation of dialysis were determined by calculating the area under the receiver operating characteristic curve $\left(A U C_{R O C}\right)$. Because CAI was classified in more than 2 groups, we used the multiclass $\mathrm{AUC}_{\mathrm{ROC}}$ as defined by Hand and Till [10]. The optimal cutoff values for intensity and percentage of each marker were defined using the Youden Index [11], which maximizes the sensitivity and specificity of the predictor. Negative predictive values (NPVs) and positive predictive values (PPVs) were calculated for each group of CAI and also for the "initiation of dialysis." All analysis was conducted at an overall significance level of 5\%. All statistical analysis was conducted using R Statistical Software version 3.2.5.

\section{RESULTS}

In the glomeruli, a higher intensity of AA (mean 1.8) was detected when glomerulosclerosis was between $26 \%$ and $50 \%$ (vs glomerulosclerosis $\leq 25 \%$, with an AA mean intensity of 1.6 , and vs glomerulosclerosis $>50 \%$, with an AA mean intensity of 1.0; Fig 1A). Cases without glomeruli at biopsy re-evaluation ( 3 cases in the group of patients with glomerulosclerosis $\leq 25 \%$ and 2 cases in the group of 


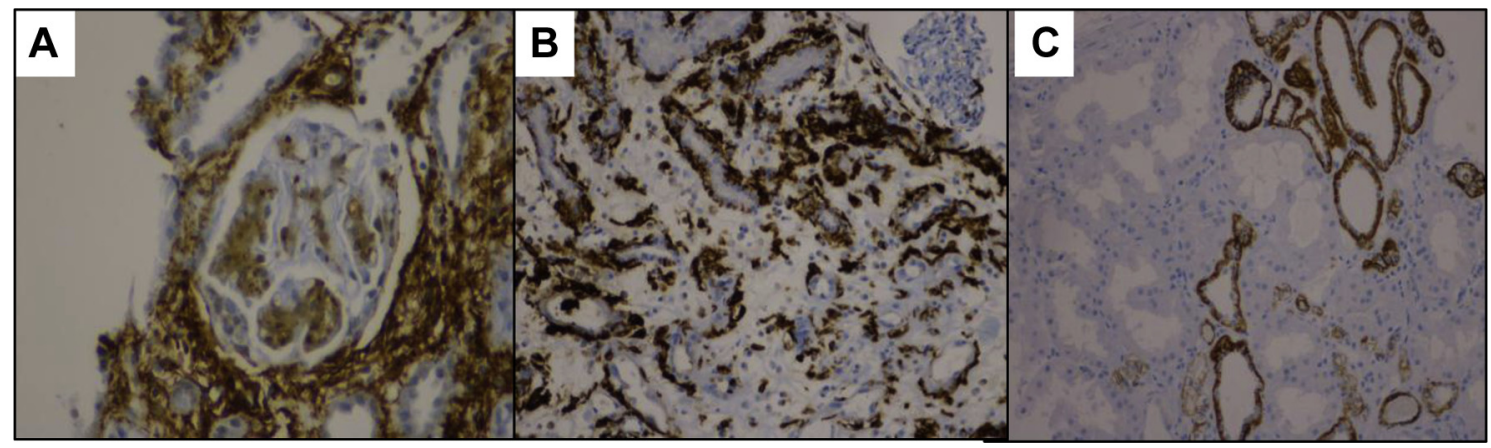

Fig 1. Biomarkers expression in the glomerulus and tubulointerstitial compartment. Alpha-smooth muscle actin-marked cells $(\times 400)$ (A) and CD163-marked cells ( $\times 200)(B)$; E-cadherin loss in more than $50 \%$ of the tubules $(\times 200)(\mathbf{C})$.

patients with glomerulosclerosis $>50 \%$ ) were excluded. The same pattern occurred with CD163, where a higher intensity (mean 2.8) was found in the group of patients with glomerulosclerosis between $26 \%$ and $50 \%$ (vs glomerulosclerosis $\leq 25 \%$, with a CD163 mean intensity of 2.7 , and vs glomerulosclerosis $>50 \%$, with a CD163 mean intensity of 2.0; Fig 1B). The percentage of AA increased as glomerulosclerosis occupied less glomerulus area. Counter to what was expected, the results did not reveal a higher percentage of AA- or CD163-marked cells in the cases with higher glomerulosclerosis.

In the tubulointerstitial compartment, the intensity or percentage of AA-marked cells was not higher in advanced interstitial fibrosis $(60.0 \%$ for interstitial fibrosis $\leq 25 \%$ group and $35.0 \%$ for interstitial fibrosis $>50 \%$ group), also counter to what was expected. A higher CD163 intensity (mean 3.0) and percentage of marked cells (mean 30.0\%) were detected in advanced interstitial fibrosis (vs interstitial fibrosis $\leq 25 \%$, with a CD163 mean intensity of 2.5 and a percentage of marked cell of $17.1 \%$, and interstitial fibrosis between $26 \%$ and $50 \%$, with a CD163 mean intensity of 2.5 and a percentage of marked cell of $24.4 \%$ ). A higher intensity or percentage of E-cadherin loss in advanced interstitial fibrosis was not detected $(55.0 \%$ for interstitial fibrosis $\leq 25 \%$ group and $40.0 \%$ for interstitial fibrosis $>50 \%$ group; Fig $1 \mathrm{C}$ ).

Furthermore, although the results expressed in Table 1 suggested a high CD163 and E-cadherin loss intensity and

Table 1. Intensity and Percentage of Marked Cells According to GFR at Biopsy Re-evaluation

\begin{tabular}{llcc}
\hline & \multicolumn{3}{c}{ GFR at the Time of Biopsy (mL/min) } \\
\cline { 2 - 4 } & $<15$ (3 Patients) & $15-29(12$ Patients) & $30-60$ (7 Patients) \\
\hline AA & & & \\
$\quad$ Glomeruli & $1+(8.3 \%)$ & $2+(13.0 \%)$ & $2+(14.0 \%)$ \\
TI & $2+(19.5 \%)$ & $2+(32.0 \%)$ & $2+(32.5 \%)$ \\
CD163 & $3+(10.0 \%)$ & $3+(5.9 \%)$ & $3+(5.6 \%)$ \\
Glomeruli & $3+(20.0 \%)$ & $3+(20.2 \%)$ & $3+(14.3 \%)$ \\
TI & $2+(60.0 \%)$ & $1+(47.1 \%)$ & $1+(56.4 \%)$ \\
E-cadherin loss & $2+(5)$
\end{tabular}

Abbreviations: AA, alpha-smooth muscle actin; GFR, glomerular filtration rate;

$\mathrm{TI}$, tubulointerstitial compartment. percentage of marked cells for lower GFR, we only found a statistically significant negative correlation between the intensity of the E-cadherin loss and the GFR $(r=-0.56$, $P=.012$; Table 2 ). A value of E-cadherin loss intensity equal or greater than 2 was obtained as cutoff for CAI group GFR $<15 \mathrm{~mL} / \mathrm{min}$ (NPV 0.95, PPV 1.0). The cutoff value for intensity of E-cadherin loss obtained for the other CAI groups was 1 , which achieved in both cases a NPV of 1 and a PPV of 0.5 for GFR between 15 and $29 \mathrm{~mL} / \mathrm{min}$ and 0.32 for GFR between 30 and $60 \mathrm{~mL} / \mathrm{min}$.

There were small differences in intensity and percentage of marked cells between the group that started dialysis and the group that did not start dialysis (Table 3). However, we identified a statistically significant association between the intensity of AA in the tubulointerstitial compartment and the initiation of dialysis $(P=.003$; Table 2$)$. A value of AA intensity in the tubulointerstitial compartment equal or

Table 2. AA, CD163, and E-Cadherin Loss Intensity/Percentage Association With Chronic Allograft Dysfunction and Initiation of Dialysis

\begin{tabular}{|c|c|c|c|}
\hline & \multicolumn{2}{|c|}{ Chronic Allograft Dysfunction } & \multirow{2}{*}{ 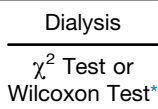 } \\
\hline & $\begin{array}{l}\text { Gamma } \\
\text { Statistic }\end{array}$ & $\begin{array}{l}\text { Spearman } \\
\text { Correlation }\end{array}$ & \\
\hline \multicolumn{4}{|l|}{ AA glomeruli } \\
\hline Intensity & 0.61 & $0.40(0.104)$ & .377 \\
\hline Percentage (\%) & 0.13 & $0.32(0.191)$ & .785 \\
\hline \multicolumn{4}{|l|}{ AA TI } \\
\hline Intensity & -0.84 & $-0.37(0.086)$ & $.003^{\dagger}$ \\
\hline Percentage (\%) & 0.23 & $0.20(0.363)$ & .506 \\
\hline \multicolumn{4}{|l|}{ CD163 glomeruli } \\
\hline Intensity & 0.24 & $0.13(0.617)$ & .056 \\
\hline Percentage (\%) & -0.53 & $-0.25(0.326)$ & .358 \\
\hline \multicolumn{4}{|l|}{ CD163 TI } \\
\hline Intensity & -1 & $-0.29(0.194)$ & $>.999$ \\
\hline Percentage (\%) & -0.26 & $-0.26(0.246)$ & $>.999$ \\
\hline \multicolumn{4}{|l|}{ E-cadherin loss } \\
\hline Intensity & -1 & $-0.56(0.012)^{\dagger}$ & $\mathrm{NE}$ \\
\hline Percentage (\%) & 0.04 & $0.04(0.852)$ & .415 \\
\hline
\end{tabular}

Abbreviations: AA, alpha-smooth muscle actin; NE, not estimable; TI, tubulointerstitial compartment.

*Intensity was assessed by $\chi^{2}$ test, and percentage was assessed by Wilcoxon test.

${ }^{\dagger}$ Significant at the $5 \%$ alpha level. 
Table 3. Intensity and Percentage of Marked Cells in the Group of Patients Who Started Dialysis and in the Group of Patients Who Did Not Start Dialysis After Kidney Allograft Biopsy

\begin{tabular}{lll}
\hline & $\begin{array}{c}\text { Dialysis } \\
\text { Patients }(\mathrm{n}=12)\end{array}$ & $\begin{array}{c}\text { Nondialysis } \\
\text { Patients }(\mathrm{n}=10)\end{array}$ \\
\hline AA & & \\
$\quad$ Glomeruli & $2+(13.0 \%)$ & $1+(12.0 \%)$ \\
TI & $2+(23.5 \%)$ & $1+(25.0 \%)$ \\
CD163 & & \\
Glomeruli & $3+(4.1 \%)$ & $2+(6.6 \%)$ \\
TI & $3+(17.0 \%)$ & $3+(20.0 \%)$ \\
E-cadherin loss & $2+(49 \%)$ & $2+(55 \%)$ \\
\hline
\end{tabular}

Abbreviations: AA, alpha-smooth muscle actin; TI, tubulointerstitial compartment.

greater than 2 of was obtained as cutoff for initiation of dialysis (NPV 0.82, PPV 0.92).

Due to the small number of patients, all the computed $95 \%$ confidence intervals for the $\mathrm{AUC}_{\mathrm{ROC}}$ to assess the overall performance of each marker in predicting CAI were too wide to have informative value for the analysis. The $\mathrm{AUC}_{\mathrm{ROC}}$ values varied between 0.409 and 0.722 for the predictors of CAI and between 0.396 and 0.867 for the predictors of initiation of dialysis. The $\mathrm{AUC}_{\mathrm{ROC}}$ value for the intensity of the E-cadherin loss in predicting CAI was 0.722 , and the $\mathrm{AUC}_{\mathrm{ROC}}$ value for the intensity of $\mathrm{AA}$ in the tubulointerstitial compartment in predicting the initiation of dialysis was 0.867 (95\% confidence interval, 0.719-1.0; Fig 2).

\section{DISCUSSION}

In the last 20 years, the literature published on new fibrogenesis markers and their effect on the kidney disease progression has been increasing. This has contributed to a better understanding

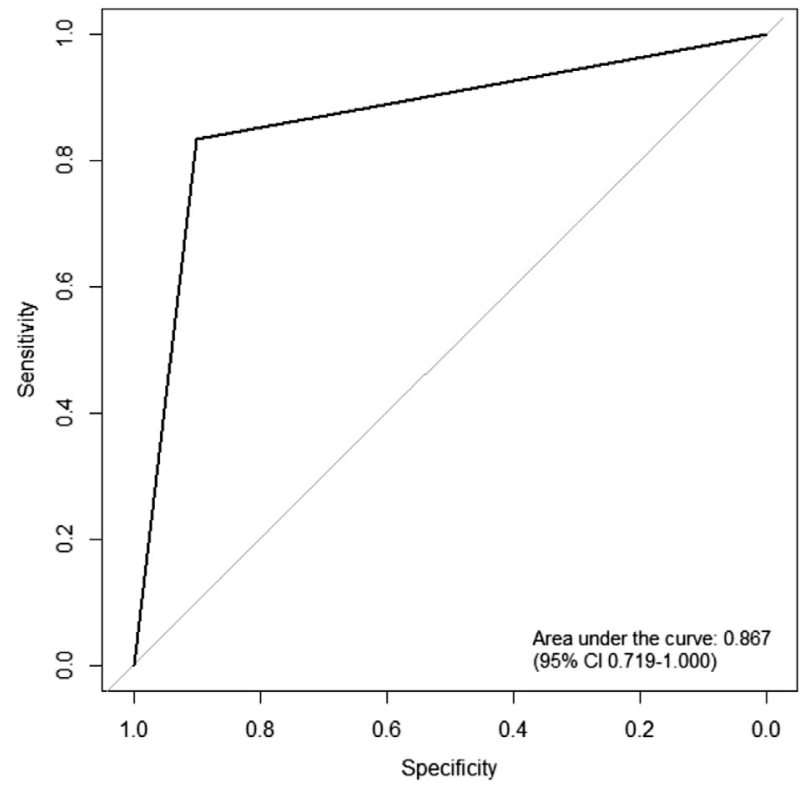

Fig 2. Receiver operating characteristic curve for the intensity of alpha-smooth muscle actin in the tubulointerstitial compartment in predicting the initiation of dialysis. Abbreviation: $\mathrm{Cl}$, confidence interval. of the fibrogenesis process and the cells and cytokines involved. Consequently, the investigation of therapeutic targets has also improved. One of these targets is the bone morphogenetic protein-7, which is expressed in the developing kidney in embryos as well as in the mature organ of adults, determining the final number of nephrons [12]. Experimental animal models have suggested that treatment with bone morphogenetic protein-7, a natural TGF- $\beta 1$ antagonist, can protect from fibrosis progression and even accelerate the return of renal function [13]. Also rapamycin and sirolimus were reported to have a significant potential for attenuating tubulointerstitial damage in rat models and may delay the progression of CAI [5]. Other targets have been studied such as Akt2 (with a critical role in mediating EMT) inhibition and administration of the antifibrotic hepatocyte growth factor, mostly in animal trials $[4,14]$. As far as we are aware of, no clinical effects of the studied markers have yet been found.

In this retrospective study, 3 of the most discussed markers of fibrosis were evaluated in kidney allograft biopsies from patients with CAI: the AA (a myofibroblast marker), the CD163 (a macrophage marker), and the loss of E-cadherin (a differentiated epithelium marker).

Evaluation of the intensity and percentage of AA- and CD163-marked cells or loss of E-cadherin, as fibrosis increases in the different compartments, have led to inconclusive results. An exception can be made in reference to the CD163 in the tubulointerstitial compartment, with a higher intensity and percentage of marked cells detected in the advanced interstitial fibrosis.

This study had some limitations such as the low number of biopsies and the observer-dependent evaluation of intensity and percentage of marked cell and E-cadherin loss. The fewer glomeruli present at the time of the biopsy re-evaluation could also have been a limitation to this study. Despite these limitations, this study showed a statistically significant prediction capacity of intensity of AA in the tubulointerstitial compartment for initiation of dialysis. The cutoff obtained (values of intensity equal or greater than 2) showed high predictive ability for initiation of dialysis according to the high NPV and PPV. We also found a statistically significant correlation between E-cadherin loss intensity and CAI. The cutoff for intensity of E-cadherin loss, as a predictor of CAI, presented high predictive value for the group of patients with GFR $<15 \mathrm{~mL} / \mathrm{min}$, contrary to the findings in the other groups. This finding suggests that this marker may be useful as a predictor for this specific CAI group and, therefore, for CAI progression. Prospective and larger studies are needed to evaluate the predictive value of these markers.

\section{REFERENCES}

[1] Bello A, Kawar B, El Kossi M, El Nahas M. Epidemiology and pathophysiology of chronic kidney disease. In: Floege J, Johnson RJ, Feehally J, editors. Comprehensive clinical nephrology. 4th ed. St. Louis, Mo: Elsevier/Saunders; 2015. p. 912-7.

[2] Shen B, Liu X, Fan Y, Qiu J. Macrophages regulate renal fibrosis through modulating TGF- $\beta$ superfamily signaling. Inflammation 2014;37:2076-84. 
[3] Du R, Xia L, Ning X, et al. Hypoxia-induced Bmil promotes renal tubular epithelial cell-mesenchymal transition and renal fibrosis via PI3K/Akt signal. Mol Biol Cell 2014;25:2650-9.

[4] Lan A, Zhang J, Xiao Z, et al. Akt2 is involved in loss of epithelial cells and renal fibrosis following unilateral ureteral obstruction. PLoS One 2014;8:e10541-51.

[5] Li X, Zhuang S. Recent advances in renal interstitial fibrosis and tubular atrophy after Kidney transplantation. Fibrogenesis Tissue Repair 2014;7:15.

[6] Nikolic-Paterson DJ, Wang S, Lan HY. Macrophages promote renal fibrosis through direct and indirect mechanisms Kidney Int Suppl 2014:4:34-48.

[7] Badid C, Desmouliere A, Babici D, et al. Interstitial expression of a-SMA: an early marker of chronic renal allograft dysfunction. Nephrol Dial Transplant 2002;17:1993-8.

[8] Fadili W, Allah MH, Laouad I. Chronic renal allograft dysfunction: risk factors, immunology and prevention. Arab J Nephrol Transplant 2013;6:45-50.
[9] Muthukumar T, Lee JR, Dadhania DM, Ding R, Sharma CK, Schwartz JE, et al. Allograft rejection and tubulointerstitial fibrosis in human kidney allografts: interrogation by urinary cell mRNA profiling. Transplant Rev 2014;28:145-6.

[10] Hand DJ, Till RJ. A Simple Generalisation of the Area Under the ROC Curve for Multiple Class Classification Problems. Machine Learning 2001;45:171.

[11] Youden WJ. Index for rating diagnostic tests. Cancer $1950 ; 3: 32-5$.

[12] Tsujimura T, Idei M, Yoshikawa M, et al. Roles and regulation of bone morphogenetic protein-7 in kidney development and diseases. World J Stem Cells 2016;8:288-96.

[13] Morrissey J, Hruska K, Guo G, et al. Bone morphogenetic protein-7 improves renal fibrosis and accelerates the return of renal function. J Am Soc Nephrol 2002;13:S14-21.

[14] Hills CE, Squires PE. The role of TGF- $\beta$ and epithelial-to mesenchymal transition in diabetic nephropathy. Cytokine Growth Factor Rev 2011;22:131-9. 